\title{
Modeling the Effect of Pathogenic Mutations on the Conformational Landscape of Protein Kinases
}

\author{
Giorgio Saladino $^{1}$, Francesco Luigi Gervasio ${ }^{1,2}$ \\ ${ }^{1}$ Department of Chemistry, University College London, London WC1E 6BT, United Kingdom \\ ${ }^{2}$ Research Institute of Structural and Molecular Biology, University College London, London \\ WC1E 6BT, United Kingdom \\ f.1.gervasio@ucl.ac.uk
}

\begin{abstract}
Most proteins assume different conformations to perform their cellular functions. This conformational dynamics is physiologically regulated by binding events and post-translational modifications, but can also be affected by pathogenic mutations. Atomistic molecular dynamics simulations complemented by enhanced sampling approaches are increasingly used to probe the effect of mutations on the conformational dynamics and on the underlying conformational free energy landscape of proteins. In this short review we discuss recent successful examples of simulations used to understand the molecular mechanism underlying the deregulation of physiological conformational dynamics due to non-synonymous single point mutations. Our examples are mostly drawn from the protein kinase family.
\end{abstract}

\section{Introduction}

Proteins are dynamic macromolecules and their function is often dependent on their motions and conformational flexibility [1]. Recent high-resolution structural studies using X-ray crystallography, Cryo-EM and NMR have given direct evidences of how protein plasticity and dynamic behavior is crucial for their function [2-4]. This is particularly true in signaling proteins such as protein kinases that react to allosteric stimuli such as ligand or protein binding and posttranslational modifications by switching to an active conformation and starting a signaling cascade that eventually controls the activity and fate of cells $[5,6]$. The current consensus is that the conformational switch is made possible due to an ensemble of different conformations being accessible to the protein, which moves on a complex conformational landscape $[7,8]$.

The substitution of one or more amino acids of a protein due to pathogenic non-synonymous mutations, may affect the conformational free energy (FE) landscape and alter the equilibrium between different conformations in an analogous way to physiological allosteric signals. Thus, mutations can also have an impact on the conformational transitions necessary for protein-protein interactions and small molecule binding [7-10]. Due to these dramatic effects, even single-point non-synonymous mutations of signaling proteins can lead to the development of several diseases and are often associated with cancer [11,12]. In this respect, understanding how mutations affect the conformational landscape of proteins (and their function) is an important step in predicting the effect of genetic mutations arising from genome-wide screening and in designing effective personalized therapies of complex multi-factorial diseases [13].

Oncogenic and drug-resistant mutations may affect the equilibria of the various conformations assumed by these proteins [17], leading to a global change in the conformational dynamics that is not easily captured by static crystal structures [18]. Atomistic molecular dynamics (MD) simulations, which are able to fully capture the dynamical nature of the proteins, are thus an increasingly useful tool in complementing structural data and understand the effect of the mutations on the conformational free energy landscape. As they provide a full atomistic description of the 
system and its dynamics, MD have been frequently described as a computational microscope [1417]. However, MD have been limited by the accessible timescales. Typically an MD simulation lasts up to a few microseconds. This issue, which is commonly known as the "timescale problem", prevents a statistically meaningful observation of conformational changes in conventional MD. Recently, the development of special-purpose hardware, such as Anton [18,19], and of new algorithms specifically aimed at enhancing the sampling of MD and solving the time-scale problem, made it possible to analyze in great detail the effects of mutations on the conformational landscape of biomolecules. An in-depth review of enhanced-sampling MD techniques is beyond the scope of the current review. In the reconstruction of conformational free energy landscapes of biomolecules, four types of algorithms (alone or in combination) are often used: 1) methods based on multiple replicas of the system such as parallel tempering or Hamiltonian replica exchange $[20,21]$; 2) methods based on the reconstruction of the free energy profile along an optimal path connecting different states, such as milestoning [22], transition path sampling [23] or the path collective variables [24]; 3) methods that enhance the sampling and reconstruct the free energy profile along a set of relevant coordinates (collective variable or CV) such as umbrella sampling [25], Targeted Molecular Dynamics (TMD)[26], Metadynamics and its many derivatives [24,25]; 4) swarms of MD trajectories and Markov State Models [27]. Here we report on recent success in the molecular understanding of the effect of pathogenic mutations on the conformational landscape of protein kinases (one of the most important and studied drug target families) through long or enhanced-sampling atomistic MD simulations.

\section{Oncogenic Mutations}

The regulatory proteins encoded by oncogenes and tumor suppressor genes play a fundamental role in the onset and progression of cancer [28]. Simulations helped the understanding of the molecular mechanism of oncogenic mutations in these crucial proteins, clarifying their mode of action in oncoproteins, such as Ras [29] and various protein kinases and in tumor suppressor genes such as p53 [28].

A prominent example is the Epidermal Growth Factor Receptor (EGFR), a tyrosine kinase that, due to its role in cancer, is one of the most studied signaling proteins . EGFR is a cell-surface receptor involved in the regulation of key cellular processes [30]. Single-point mutations in EGFR, such as L858R (see Figure 1) are among the most frequently observed in lung carcinoma [31]. In-vitro experiments appear to suggest that EGFR mutants are more active than the wild type [32,33] Several computational studies have addressed the molecular mechanism by which these mutations affect the complex EGFR regulation, complementing the abundant experimental data. Dixit and Verkhivker [26] simulated the activation of EGFR WT and of the L858R mutant. During activation, in EGFR, as in most protein kinases, the long activation loop (A-loop) assumes an extended configuration (see Fig. 1). The authors used TMD to shift the loop from the inactive to the active conformations. In the simulations, they observed a two-step mechanism. First the functionally important $\alpha \mathrm{C}$-helix is repositioned to assume an active-like conformation. 

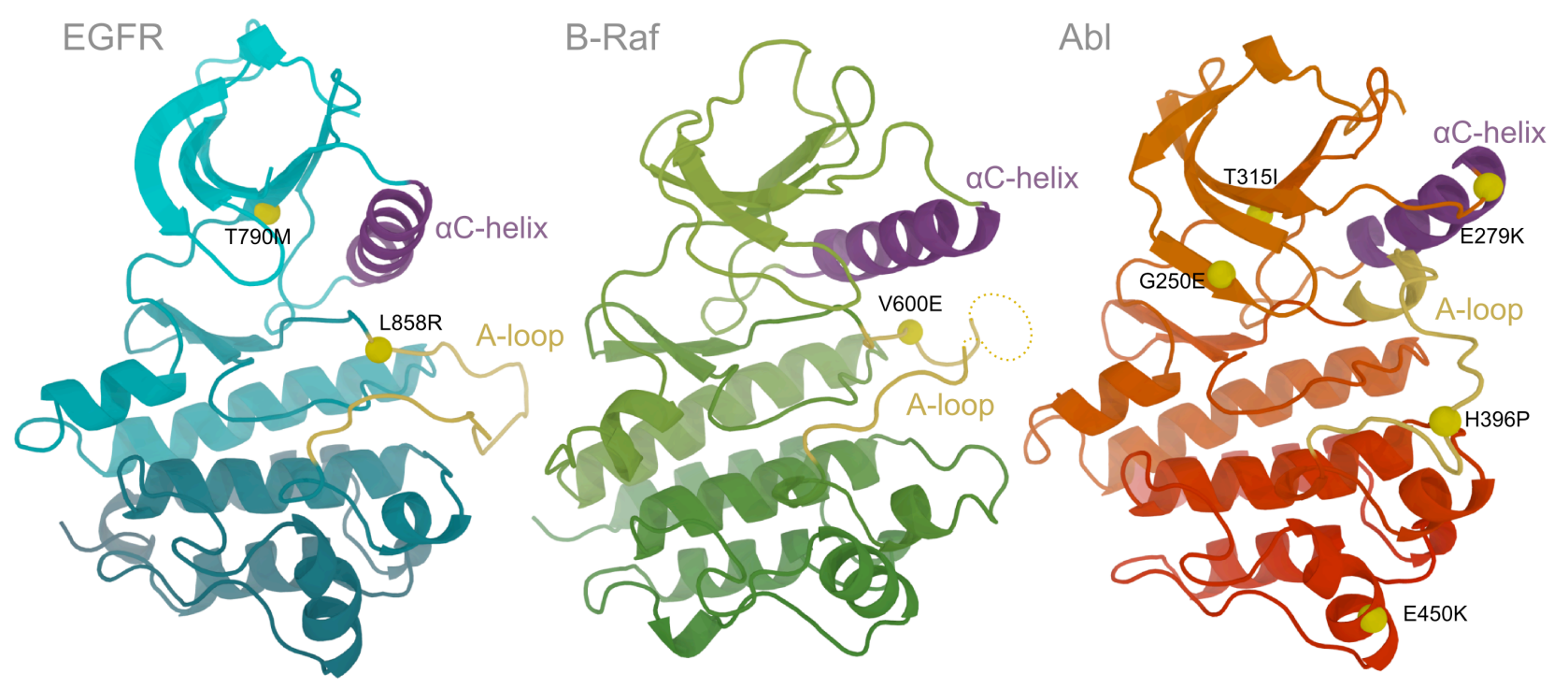

Figure 1. The structure of EGFR (cyan, active state), B-Raf (green, semi-active) and Abl (red, inactive) are shown with oncogenic and drug resistant mutations indicated by yellow spheres. The A-loop is open and fully extended in the active structure and closed in the inactive one. The important $\alpha \mathrm{C}$-helix is highlighted in purple.

The authors concluded that the mutation appear to stabilize the active state, by aiding the assembling of the so-called hydrophobic spine, and the transition of the $\alpha \mathrm{C}$-helix. Wan and Coveney [34] performed long multiple-replica MD simulations of wild-type and L858R EGFR starting from active and inactive crystal structures. They observed that, while both the A-loop and the $\alpha \mathrm{C}$-helix have fluctuations similar to the rest of the protein in the active state, their mobility changes significantly in the inactive one: the A-loop appears more flexible, while the $\alpha \mathrm{C}$ is considerably more rigid. Analyzing the distribution of orientations of the $\alpha \mathrm{C}$-helix, the authors also discovered large orientational rearrangements in the active state. L858R changes this distribution and generates alternative orientations of the helix that increase the cavity between the kinase two lobes, thus easing the A-loop transition by removing sterical constraints. In agreement with previous reports, the authors suggest that while the inactive state of EGFR is energetically favored, L858R disturbs the equilibrium and favors the inactive-to-active transition, while promoting the appearance of intermediate states with different orientations of the $\alpha \mathrm{C}$-helix.

Using the special-purpose computer Anton $[18,19]$ Shan and co-workers were able to assess the effect of L858R mutations with very long MD simulations [35]. The authors found that, starting from the active structure, the $\alpha \mathrm{C}$ helix tends to shift to the inactive $\alpha \mathrm{C}$ conformation ( $\alpha \mathrm{C}$-out). Moreover, they observed that the transition is always accompanied by a loss of structural integrity of the $\alpha \mathrm{C}$-helix. The partial disorder of this region is confirmed by $\mathrm{X}$-ray structures showing unresolved and high B-factor residues in this area. Accordingly, they propose a three-state energy landscape for the EGFR monomeric form in which the active state of EGFR is marginally stable and the newly discovered disordered state is the most favorable one, followed by the inactive structure. As EGFR full activation is achieved only upon dimerization, when the large lobe of an "activator" protein interacts with the small lobe of a "receiver" kinase, they also suggest that ordering of the $\alpha \mathrm{C}$ region, located at the EGFR receiver interface, might occur upon dimerization. Interestingly, simulating the asymmetric EGFR dimer, the authors were able to show that the ordering of the interface region stabilizes the dimer complex. When simulating the L858R mutant, Shan and coworkers found that the transition from the $\alpha \mathrm{C}$-in active conformation to the $\alpha \mathrm{C}$-out still happens, but is considerably slower. As proposed in Ref. [26], the authors also reported that stabilization of the $\alpha \mathrm{C}$-in active conformation is the main effect of the Leu858 substitution. Comparing the simulations with H/D experiments, they also concluded that the stabilization of the 
active structure is at the expenses of the disordered state, which agrees with the higher propensity of L858R to form dimers. Notwithstanding the remarkable achievements of long MD smimulations, the lack of sufficient sampling prevented the reconstruction of the complete conformational free energy landscape of EGFR.

This was addressed by Sutto and Gervasio [36], who used a large-scale Parallel Tempering Metadynamics [37] (PTMetaD) simulation, a combination of metadynamics [38,39] and Parallel Tempering [20] (PT). The computed free energy landscape of EGFR (see Figure 2) shows that the most populated basin in the WT corresponds to the inactive (Src-like) structure, with the A-loop folded onto itself in a closed conformation. A basin with an $\alpha \mathrm{C}$-helix partially disordered, as observed by Shan et al., is also populated. In agreement with previous reports, the active state was infrequently visited during the simulations of EGFR WT. Interestingly, the authors observed that the L858R mutant populates the active state, even if the most stable state is represented by a "semiclosed" ensemble of conformations, somehow in-between the active and inactive states. In agreement with Shan et al., a suppression of the $\alpha \mathrm{C}$-helix disorder, aiding the formation of the dimer, is also reported.
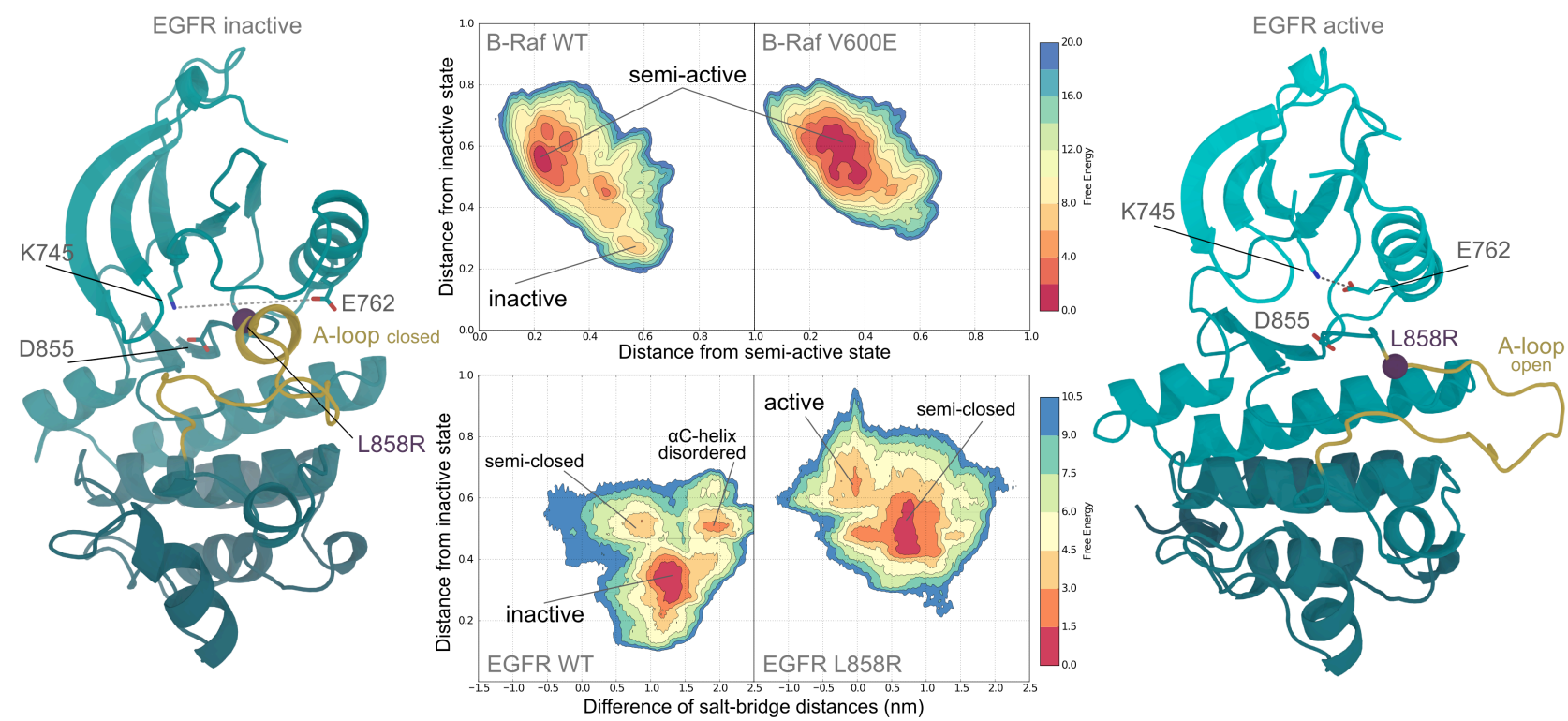

Figure 2. Free energy surfaces for the active-to-inactive transition of EGFR and B-Raf. The inactive (left, A-loop closed) and active (right, A-loop open) of EGFR are reported for reference. The salt-bridges used as collective variable in EGFR are reported onto the two structures. Oncogenic mutations (as L858R, shown with a purple sphere) shift the population towards more active-like structures.

The free energy landscapes reported in Ref. [36], thus, seem to reconcile the results reported in the literature for which several different intermediates, with different orientations and partial disorder of the $\alpha \mathrm{C}$-helix, were observed.

The importance of the transitions of the $\alpha \mathrm{C}$-helix from the inactive "out" conformation to the active $\alpha \mathrm{C}$-helix "in" conformation and its stability was also confirmed by a recent paper by Ruan \& Kannan [40] who combined cell-based assays with simulations to study the effect of the oncogenic $\mathrm{R} 776 \mathrm{H}$ mutant. They report that the $\mathrm{R} 776 \mathrm{H}$ mutant increases affinity for dimerization by stabilizing the $\alpha \mathrm{C}$-helix of the acceptor protomer in an "in" conformation.

Albeit fully converged free energy landscapes of WT and mutant proteins provide a quantitative and exhaustive picture of the effect of the mutations, they require lengthy and expensive simulations. A promising alternative is to run a few standard MD trajectories to probe the local dynamics and complement the information with an analysis of the protein energetics. With this 
approach it was possible to relate the flexibility changes observed in the EGFR G719S oncogenic mutant with specific functional effects [41].

Another oncogenic kinase of great biomedical interest due to its involvement in melanoma is BRaf. By using enhanced-sampling MD simulations, Marino et al. [42] recently described the effect of a widespread cancer-causing mutation in the B-Raf kinase domain. The authors run extensive PTmetaD simulations, $1.9 \mu$ s for each of the 36 replicas used, of the WT and the V600E mutant (see Figure 1), a mutation often associated to melanoma. From the reconstructed free energy surfaces (see Figure 2) the authors found that, at odds with what observed for EGFR [36], the most stable free energy basin for WT B-Raf does not correspond to an inactive-like state. The representative structures appear to have an A-loop in an intermediate state that was termed "semiactive". The inactive ( $\alpha \mathrm{C}$-out) conformation is also explored in the WT simulation. These observations are in agreement with the reported importance of the asymmetric dimer formation in the full activation of WT B-Raf $[43,44]$. The B-Raf V600E mutant, which is known to be less dependent on dimerization to be fully active, is predicted to have an effect on the conformational free energy landscape similar to the one observed for the EGFR oncogenic mutants [42]. V600E shifts the equilibrium towards the active-like structure, increasing even further the energy penalty of the inactive one.

In a related paper Jambrina et al. used Markov state models to quantify the effect of phosphorylation of the N-terminal acidic (NtA) motif on RAF homo- and hetero- dimerization [45]. Their extensive analysis, corroborated by experimental evidence, shows the importance of NtA phosphorylation on the stability of the dimer and explain the importance of a conserved Trp residue (W450) on transactivation and dimer formation.

Finally we will discuss the interesting mode of action of a common oncogenic mutation of phosphatidylinositol 3-kinase alpha (PI3Ka), H1047R, which results in enzymatic over-activation. By using a combination of MD simulations and experiments it was shown that the mutation abolishes the auto-inhibitory role of the C-terminal tail in the protein, enhances protein-membrane binding, and changes the conformation of His917, the key residue for hydrolysis [46].

\section{Drug Resistant Mutations}

The emergence of drug resistant mutations is one of the main challenges in the treatment of several diseases. Understanding how a drug-resistant mutation interferes with the binding of drugs, without adversely affecting its function is thus of great importance. Computational studies have been devoted to understanding the effect of drug resistant mutations on proteins implicated in several diseases, ranging from HIV protease [47,48] and reverse transcriptase [49] to porins in bacteria [50], neuraminidase in influenza virus [51] and protein kinases in cancer. The changes to the conformational landscape caused by drug resistance mutations were recently analyzed in great details for the Abl tyrosine kinase [10] (see Figure 2). A chromosomal translocation, originating the infamous Philadelphia chromosome, leads to the over-activation of Abl in chronic myeloid leukemia (CML) [52,53]. The discovery of the potent inhibitor Imatinib revolutionized the treatment of CML [54]. Unfortunately, the emergence of drug resistant mutation in Abl can render the drug ineffective. While some mutations are located near the drug-binding site and prevent the binding of Imatinib, several others are located in distant regions of the catalytic domain and act allosterically. Lovera and coworkers [10], who have previously proposed that Imatinib specificity in different kinases is due to the different stability of the inactive, so-called "DFG-out", conformation [55], studied five Abl mutants whose mechanism of action is unknown, or debated. Using long Parallel Tempering Metadynamics [37] simulations, the authors were able to describe the complex changes to the free energy landscape, affecting two important conformational transition in protein kinases. Indeed, not only do the mutations affect the equilibrium between the inactive "DFG-out" conformation, that binds Imatinib, and the main inactive conformation ("DFG- 
in"), but also the reciprocal stability of the activation loop open and closed conformations. In particular, the simulations revealed that for some mutants, like H396P and E279K, the "DFG-out" conformation is significantly destabilized, thus changing the affinity of the drug. Other mutations, however, as the famous "gatekeeper" mutation T315I [56], have little effect on the DFG transition and, instead, mainly act on equilibrium between the inactive structure(s) and the active one and have an effect on the binding kinetics by changing the dynamics of the G-loop. By favoring the Aloop open, active-like, structure, the mutations still reduce the population of Abl molecules able to bind Imatinib. A similar effect of the A-loop conformation was also observed in EGFR [36] and in FGFR [57]. The T790M mutation in EGFR (see Figure 1), which is the analogous of the Abl "gatekeeper" mutation, was shown to have a dramatic effect on the free energy landscape, stabilizing the active A-loop open state and to have some degree of cooperativity with the L858R mutation. Similarly, the V561M gatekeeper mutation was shown to have a significant effect on the conformational energy landscape of FGFR1, another receptor tyrosine kinase playing an important role in cancer [57]. The computed free energy surfaces show a significant population shift towards an active-like extended A-loop conformations.

Conclusions. Non-synonymous single-point mutations often have a significant effect on the conformational free energy landscape of regulatory proteins such as protein kinases. Atomistic MD simulations complemented by enhanced sampling algorithms, free energy methods and energetic analysis are an increasingly important and powerful tool in understanding the molecular mechanisms underlying such changes and in quantifying the effect of the changes in excellent agreement with experimental results. These methods are thus able to complement the crystallographic and spectroscopic results in a number of ways and will most probably play a prominent role in predicting the effect of mutations in personalized medicine.

Acknowledgements. This work was supported by the Engineering and Physical Sciences Research Council [Grant EP/M013898/1].

\section{References}

1. Karplus M, Kuriyan J: Molecular dynamics and protein function. Proc. Natl. Acad. Sci. U. S. A. 2005, 102:6679-85.

2. Fischer N, Konevega AL, Wintermeyer W, Rodnina M V., Stark H: Ribosome dynamics and tRNA movement by time-resolved electron cryomicroscopy. Nature 2010, 466:329333.

3. Tzeng SR, Kalodimos CG: Protein dynamics and allostery: An NMR view. Curr. Opin. Struct. Biol. 2011, 21:62-67.

4. Yang H, Luo G, Karnchanaphanurach P, Louie T-M, Rech I, Cova S, Xun L, Xie XS:

Protein conformational dynamics probed by single-molecule electron transfer. Science (80-. ). 2003, 302:262-266.

5. Cohen P: Protein kinases - the major drug targets of the twenty-first century? Nat. Rev. Drug Discov. 2002, 1:309-315.

6. Cohen P: The origins of protein phosphorylation. Nat. Cell Biol. 2002, 4:E127-E130.

7. Tsai C-J, Nussinov R: A unified view of "how allostery works". PLoS Comput. Biol. 2014, 
10:e1003394. (.*) "A perspective on the numerous studies and theories involving allostery and its connection to structural ensembles. A comprehensive view is developed that addresses the thermodynamics and structural determinant of allosteric communication."

8. Miyashita O, Onuchic JN, Wolynes PG: Nonlinear elasticity, proteinquakes, and the energy landscapes of functional transitions in proteins. Proc. Natl. Acad. Sci. USA 2003, 100:12570-12575.

9. Winkler DG, Johnson JC, Cooper JA, Vojtek AB: Identification and Characterization of Mutations in Ha-Ras That Selectively Decrease Binding to cRaf-1. J. Biol. Chem. 1997, 272:24402-24409.

10. Lovera S, Morando M, Pucheta-Martinez E, Martinez-Torrecuadrada JL, Saladino G, Gervasio FL: Towards a Molecular Understanding of the Link between Imatinib Resistance and Kinase Conformational Dynamics. PLoS Comput Biol 2015, 11:e1004578. (.*) "A comprehensive study on the effect of drug resistant mutations on the conformational landscape of Abl tyrosine kinase."

11. Futreal PA, Coin L, Marshall M, Down T, Hubbard T, Wooster R, Rahman N, Stratton MR: A census of human cancer genes. Nat. Rev. Cancer 2004, 4:177-183.

12. Stratton MR, Campbell PJ, Futreal PA: The cancer genome. Nature 2009, 458:719-724.

13. Saladino G, Gervasio FL: New Insights in Protein Kinase Conformational Dynamics. Curr. Top. Med. Chem. 2012, 12:1889-1895. (·) "Computational studies aimed at furthering the understanding of how conformational transition are involved in protein kinase regulation are reviewed."

14. Lee EH, Hsin J, Sotomayor M, Comellas G, Schulten K: Discovery through the computational microscope. Structure 2009, 17:1295-306.

15. Sotomayor M, Schulten K: Single-molecule experiments in vitro and in silico. Science 2007, 316:1144-1148.

16. Dror RO, Dirks RM, Grossman JPP, Xu H, Shaw DE: Biomolecular simulation: a computational microscope for molecular biology. Annu. Rev. Biophys. 2012, 41:429-52.

17. Malmstrom RD, Kornev AP, Taylor SS, Amaro RE: Allostery through the computational microscope: cAMP activation of a canonical signalling domain. Nat. Commun. 2015, 6:7588.

18. Shaw DE, Bowers KJ, Chow E, Eastwood MP, Ierardi DJ, Klepeis JL, Kuskin JS, Larson RH, Lindorff-Larsen K, Maragakis P, et al.: Millisecond-scale molecular dynamics simulations on Anton. In Proceedings of the Conference on High Performance Computing Networking, Storage and Analysis - SC ’09. ACM Press; 2009:1. 
19. Shaw DE, Grossman JP, Bank JA, Batson B, Butts JA, Chao JC, Deneroff MM, Dror RO, Even A, Fenton $\mathrm{CH}$, et al.: Anton 2: Raising the Bar for Performance and Programmability in a Special-Purpose Molecular Dynamics Supercomputer. In SC14: International Conference for High Performance Computing, Networking, Storage and Analysis. 2014:41-53.

20. Hansmann UHE: Parallel tempering algorithm for conformational studies of biological molecules. Chem. Phys. Lett. 1997, 281:140-150.

21. Sugita Y, Okamoto Y: Replica-exchange multicanonical algorithm and multicanonical replica-exchange method for simulating systems with rough energy landscape. Chem. Phys. Lett. 2000, 329:261-270.

22. West AM a, Elber R, Shalloway D: Extending molecular dynamics time scales with milestoning: example of complex kinetics in a solvated peptide. J. Chem. Phys. 2007, 126:145104.

23. Bolhuis PG, Chandler D, Dellago C, Geissler PL: Transition path sampling: throwing ropes over rough mountain passes, in the dark. Ann. Rev. Phys. Chem. 2002, 53:291-318.

24. Branduardi D, Gervasio FL, Parrinello M: From A to B in free energy space. J. Chem. Phys. 2007, 126:054103.

25. Torrie GM, Valleau JP: Nonphysical sampling distributions in Monte Carlo free-energy estimation: umbrella sampling. J. Comp. Phys. 1977, 23:187-199.

26. Dixit A, Verkhivker GM: Hierarchical modeling of activation mechanisms in the ABL and EGFR kinase domains: thermodynamic and mechanistic catalysts of kinase activation by cancer mutations. PLoS Comput. Biol. 2009, 5:e1000487.

27. Chodera JD, Noé F: Markov state models of biomolecular conformational dynamics. Curr. Opin. Struct. Biol. 2014, 25:135-144.

28. Steen HB: The origin of oncogenic mutations: where is the primary damage?. Carcinogenesis 2000, 21:1773-6.

29. Grant BJ, Gorfe AA, McCammon JA: Ras Conformational Switching: Simulating Nucleotide-Dependent Conformational Transitions with Accelerated Molecular Dynamics. PLoS Comput. Biol. 2009, 5:e1000325.

30. Roskoski R: The ErbB/HER family of protein-tyrosine kinases and cancer. Pharmacol. Res. 2014, 79:34-74.

31. Sharma S V, Bell DW, Settleman J, Haber D a: Epidermal growth factor receptor mutations in lung cancer. Nat Rev Cancer 2007, 7:169-181.

32. Yun CH, Boggon TJ, Li Y, Woo MS, Greulich H, Meyerson M, Eck MJ: Structures of 
Lung Cancer-Derived EGFR Mutants and Inhibitor Complexes: Mechanism of Activation and Insights into Differential Inhibitor Sensitivity. Cancer Cell 2007, 11:217-227.

33. Zhang X, Gureasko J, Shen K, Cole PA, Kuriyan J: An allosteric mechanism for activation of the kinase domain of epidermal growth factor receptor. Cell 2006, 125:1137-49.

34. Wan S, Coveney P V: Molecular dynamics simulation reveals structural and thermodynamic features of kinase activation by cancer mutations within the epidermal growth factor receptor. J. Comput. Chem. 2011, 32:2843-52.

35. Shan Y, Eastwood MP, Zhang X, Kim ET, Arkhipov A, Dror RO, Jumper J, Kuriyan J, Shaw DE: Oncogenic mutations counteract intrinsic disorder in the EGFR kinase and promote receptor dimerization. Cell 2012, 149:860-870. (•) "Long multi- $\mu$ s MD simulations on the special-purpose machine Anton are combined with experiments to understand the effect of mutations on the structure and dynamics of EGFR"

36. Sutto L, Gervasio FL: Effects of oncogenic mutations on the conformational free-energy landscape of EGFR kinase. Proc. Natl. Acad. Sci. U. S. A. 2013, 110:10616-21. ( •) “The conformational free energy landscape of the EGFR kinase and its mutants is obtained using parallel tempering simulations combined with Metadynamics, revealing the molecular mechanism of cancer-causing and drug-resistance-inducing mutations."

37. Bussi G, Gervasio FL, Laio A, Parrinello M: Free-energy landscape for beta hairpin folding from combined parallel tempering and metadynamics. J. Am. Chem. Soc. 2006, 128:13435-41.

38. Laio A, Parrinello M: Escaping free-energy minima. Proc. Natl. Acad. Sci. U. S. A. 2002, 99:12562-6.

39. Laio A, Gervasio FL: Metadynamics: a method to simulate rare events and reconstruct the free energy in biophysics, chemistry and material science. Rep. Prog. Phys. 2008, 71:126601.

40. Ruan Z, Kannan N: Mechanistic Insights into R776H Mediated Activation of Epidermal Growth Factor Receptor Kinase. Biochemistry 2015, 54:4216-25.

41. Paladino A, Morra G, Colombo G: Structural Stability and Flexibility Direct the Selection of Activating Mutations in Epidermal Growth Factor Receptor Kinase. $J$. Chem. Inf. Model. 2015, 55:1377-1387.

42. Marino KA, Sutto L, Gervasio FL: The Effect of a Wide-spread Cancer-causing Mutation on the Inactive to Active Dynamics of the B-Raf Kinase. J. Am. Chem. Soc. 
2015, 137:5280-5283. ( • ) "The free energy landscape for the activations of B-Raf WT and the oncogenic mutant V600E is obtained with Parallel Tempering Metadynamics. Based on the free energy landscapes, a new mode of action was proposed for the mutations reconciling different mechanisms previously proposed in the literature."

43. Jambrina PG, Bohuszewicz O, Buchete N-V, Kolch W, Rosta E: Molecular mechanisms of asymmetric RAF dimer activation. Biochem. Soc. Trans. 2014, 42:784-790.

44. Hu J, Stites EC, Yu H, Germino EA, Meharena HS, Stork PJS, Kornev AP, Taylor SS, Shaw AS: Allosteric activation of functionally asymmetric RAF kinase dimers. Cell 2013, 154:1036-46. (·) "The mechanism of activation of RAF kinases is discussed, showing the prominent role of dimerization and of the assembly of the hydrophobic spine."

45. Jambrina PG, Rauch N, Pilkington R, Rybakova K, Nguyen LK, Kholodenko BN, Buchete N-V, Kolch W, Rosta E: Phosphorylation of RAF Kinase Dimers Drives Conformational Changes that Facilitate Transactivation. Angew. Chemie Int. Ed. 2015, doi:10.1002/anie.201509272.

46. Gkeka P, Evangelidis T, Pavlaki M, Lazani V, Christoforidis S, Agianian B, Cournia Z: Investigating the structure and dynamics of the PIK3CA wild-type and H1047R oncogenic mutant. PLoS Comput. Biol. 2014, 10:e1003895.

47. Zhang J, Hou T, Wang W, Liu JS: Detecting and understanding combinatorial mutation patterns responsible for HIV drug resistance. Proc. Natl. Acad. Sci. 2010, 107:13211326.

48. Wang W, Kollman PA: Computational study of protein specificity: The molecular basis of HIV-1 protease drug resistance. Proc. Natl. Acad. Sci. 2001, 98:14937-14942.

49. Rodríguez-Barrios F, Balzarini J, Gago F: The molecular basis of resilience to the effect of the Lys103Asn mutation in non-nucleoside HIV-1 reverse transcriptase inhibitors studied by targeted molecular dynamics simulations. J. Am. Chem. Soc. 2005, 127:75707578.

50. Groot BL De, Zachariae U, Kutzner C, Grubmüller H, de Groot BL, Zachariae U: Computational electrophysiology: the molecular dynamics of ion channel permeation and selectivity in atomistic detail. Biophys. J. 2011, 101:809-17.

51. Woods CJ, Malaisree M, Pattarapongdilok N, Sompornpisut P, Hannongbua S, Mulholland AJ: Long Time Scale GPU Dynamics Reveal the Mechanism of Drug Resistance of the Dual Mutant I223R/H275Y Neuraminidase from H1N1-2009 Influenza Virus. Biochemistry 2012, 51:4364-4375.

52. Lin J, Arlinghaus R: Activated c-Abl tyrosine kinase in malignant solid tumors. 
Oncogene 2008, 27:4385-91.

53. Hantschel O, Superti-Furga G: Regulation of the c-Abl and Bcr-Abl tyrosine kinases. Nat. Rev. Mol. Cell Biol. 2004, 5:33-44.

54. Deininger M, Buchdunger E, Druker BJ: The development of imatinib as a therapeutic agent for chronic myeloid leukemia. Blood 2005, 105:2640-53.

55. Lovera S, Sutto L, Boubeva R, Scapozza L, Dölker N, Gervasio FL: The different flexibility of c-Src and c-Abl kinases regulates the accessibility of a druggable inactive conformation. J. Am. Chem. Soc. 2012, 134:2496-9. ( * ) "The different affinity of Imatinib for the tyrosine kinases $\mathrm{Abl}$ and $\mathrm{Src}$ is explained calculating the free energy landscape for the DFG-flip transition to the drug-bound conformation."

56. Gibbons DL, Pricl S, Kantarjian H, Cortes J, Quintás-Cardama A: The rise and fall of gatekeeper mutations? The BCR-ABL1 T315I paradigm. Cancer 2012, 118:293-9.

57. Bunney TD, Wan S, Thiyagarajan N, Sutto L, Williams S V., Ashford P, Koss H, Knowles M a., Gervasio FL, Coveney P V., et al.: The Effect of Mutations on Drug Sensitivity and Kinase Activity of Fibroblast Growth Factor Receptors: A Combined Experimental and Theoretical Study. EBioMedicine 2015, 2:194-204. 